\title{
AN ACCESSIBILITY PROOF OF ORDINAL DIAGRAMS IN INTUITIONISTIC THEORIES FOR ITERATED INDUCTIVE DEFINITIONS
}

By

\section{Toshiyasu ARAI}

Let $(I, \prec)$ be a non-empty well-ordered system with the least element 0 , and $\tilde{I}$ be $I \cup\{\infty\}$ with the largest element $\infty$. Let $A$ be a non-empty well-ordered set. Then $O(I, A)$ denotes the system of ordinal diagrams (o.d.'s) based on $I$ and $A$. (cf. $[9, \S 26]$.) The accessibility proof for $O(I, A)$ in $[9$, pp. 298-309] shows that every o.d. from $O(I, A)$ is accessible with respect to $<_{i}$ for every $i$ in $\tilde{I}$.

The central notions in this proof are $i$-fans and $i$-accessibility for $i$ in $\tilde{I}$. Roughly speaking, an o.d. $\mu$ is an $i$-fan if for every $j<i$ and every $j$-section $\nu$ of $\mu, \nu$ is $j$-accessible, and an o.d. is $i$-accessible if it is accessible in $i$-fans with respect to $<_{i}$.

Consider the case when the order type of $(I, \prec)$ is a successor ordinal $\xi+1$. If we formalize this accessibility proof for $O(\xi+1,1)(=O(I, 1))$ naturally, then this proof can be done in the intuitionistic theory $\operatorname{ID}_{\xi+1}^{i}$ for $\xi+1$-times iterated inductive definitions.

The purpose of this paper is to show the following fact: the accessibility of each o.d. from $O(\xi+1,1)$ with respect to $<_{0}$ is derivable in $\mathrm{ID}_{\xi}^{\mathrm{i}}$. (Theorem)

In the case when $\xi$ equals $\omega$, this theorem will complement the consistency proof in [1] in the following sense. We will give in [1] a consistency proof for the subsystem $\left(\Pi_{1}^{1}-\mathrm{CA}\right)+(\mathrm{BI})$ of classical analysis by the accessibility of $O(\omega+1,1)$ with respect to $<_{0}$. It follows from the well-known equivalence between the classical version $\mathrm{ID}_{\omega}$ of $\mathrm{ID}_{\omega}^{\mathrm{i}}$ and $\left(\Pi_{1}^{1}-\mathrm{CA}\right)+(\mathrm{BI})$ that this consistency proof is optimal.

The author is indebted to Dr. T. Yukami for the seminar under the guidance of him during the preparation of this paper. The author wishes to express his heart-felt thanks to Prof. N. Motohashi for reading this paper in manuscript and suggesting a number of linguistic improvements.

Received October 21, 1983. 
Let $<$ be a primitive recursive well-ordering with the least element 0 and the largest element $\xi$, and $I$ be the primitive recursive domain of $\prec$. Let $\lambda x . x \oplus 1$ and $\lambda x, x \ominus 1$ be primitive recursive successor and predecessor function with respect to $\prec$, respectively. And we will assume throughout this paper that the above facts except the well-orderedness of $\prec$ are all derivable in the primitive recursive arithmetic PRA, that is to say, we will assume that the following formulae are all derivable in PRA :

$$
\begin{aligned}
& x<y \longrightarrow I(x) \wedge I(y), \\
& I(x) \longrightarrow 7(x<x), \\
& x \prec y \wedge y<z \longrightarrow x<z, \\
& I(x) \wedge I(y) \longrightarrow x<y \vee x=y \vee y<x, \\
& I(0), I(x) \longrightarrow 0 \leqq x, \quad(x \leqq y:=x<y \vee x=y) \\
& I(x) \longrightarrow x \leqq \xi, \\
& I(x) \longrightarrow x \leqq x \oplus 1, \\
& x<\xi \longrightarrow x<x \oplus 1, \\
& y<x \longrightarrow y \oplus 1 \leqq x, \\
& I(x) \longrightarrow x \ominus 1 \leqq x, \\
& x<\xi \longrightarrow(x \oplus 1) \ominus 1=x, \\
& x \ominus 1<x \longrightarrow x=(x \ominus 1) \oplus 1 .
\end{aligned}
$$

Then the following formulae are also derivable in PRA:

$$
\begin{aligned}
& x<\xi \longrightarrow(y<x \oplus 1 \longleftrightarrow y \leqq x), \\
& x<\xi \longrightarrow(x \leqq y \leqq x \oplus 1 \longrightarrow y=x \vee y=x \oplus 1), \\
& y<\xi \longrightarrow(y \oplus 1=x \longrightarrow x \ominus 1<x) .
\end{aligned}
$$

Further let Suc and Lim be unary predicate constants with their defining axioms:

$$
\begin{aligned}
& \operatorname{Suc}(x) \longleftrightarrow x \ominus 1<x, \\
& \operatorname{Lim}(x) \longleftrightarrow I(x) \wedge x \neq 0 \wedge>\operatorname{Suc}(x) .
\end{aligned}
$$

Then the following formulae are also derivable in PRA:

$$
\begin{aligned}
& I(x) \longrightarrow(x=0 \vee \operatorname{Suc}(x) \vee \operatorname{Lim}(x)) . \\
& \operatorname{Lim}(x) \wedge y<x \longrightarrow y \oplus 1<x .
\end{aligned}
$$

Next, we will consider the system of o.d.'s $O^{*}(I, 1) . O^{*}(I, 1)$ is an inessential 
modification of $O(I, 1)$. In contrast with $O(I, 1), O^{*}(I, 1)$ has an identity 0 with respect to \#. For the precise definition of $O^{*}(I, 1)$, we refer to Levitz [7].

We will assume an arithmetization of the o.d.'s in $O^{*}(I, 1)$. Thus we have the following predicate constants for primitive recursive predicates:

'* is an o.d.', ' $*_{1}$ is a component of $*_{2}$,'

$*_{1} \equiv *_{2}$ for ' $*_{1}, *_{2}$ are o.d.'s and $*_{1}$ is equal to $*_{2}$. ',

$*_{1} \subset *_{3} *_{2}$ for ' $*_{1}, *_{2}$ are o.d.'s, $*_{3} \leqq \xi$ and $*_{1}$ is a $*_{3}$-section of $*_{2}$.',

$*_{1}<*_{3} *_{2}$ for $*_{1}, *_{2}$ are o.d.'s, $*_{3} \leqq \xi$ and $*_{1}$ is smaller than $*_{2}$

with respect to $<_{*_{3}}$.

In the following, we will employ the following syntactical variables:

$i, j, k$ vary through the elements in $I$,

$\mu, \nu, \rho, \lambda$ vary through o.d.'s.

Following Kreisel [6], we will define the notion of $i$-accessibility for $i \prec \xi$ in $\operatorname{ID}_{\xi}^{\mathrm{i}}(\mathfrak{A})$ for some positive operator form $\mathfrak{A}$. Let $\mathfrak{A}(X, Y, i, \mu)$ be the following positive operator form :

$$
\mathfrak{F}(i, \mu, Y) \wedge \forall \nu<_{i} \mu(\mathfrak{F}(i, \nu, Y) \longrightarrow X(\nu))
$$

where $\mathfrak{F}(i, \mu, Y)$ is the formula $\forall k\left\langle i \forall \rho \subset_{k} \mu Y(k, \rho)\right.$.

Let $\operatorname{Prog}[X, R, Y]$ be the formula

$$
\forall \mu(X(\mu) \wedge \forall \nu(R(\nu, \mu) \wedge X(\nu) \longrightarrow Y(\nu)) \longrightarrow Y(\mu)) .
$$

If we write $A$ for the set constant $P^{\mathfrak{A}}$, and $F_{i}(\mu)$ for $\forall j\left\langle i \forall \nu \subset_{j} \mu A_{j}(\nu)\right.$, then the axioms $\left(P^{\mathfrak{Y}} .1\right)_{\xi}$ and $\left(P^{\mathfrak{Y}} .2\right)_{\xi}$ in $\left[4\right.$, p. 307] become the following $(A .1)_{\xi}$ and $(A .2)_{\xi}$, respectively :

$(A .1)_{\xi} \quad \forall i<\xi \operatorname{Prog}\left[F_{i},<_{i}, A_{i}\right]$,

$(A .2)_{\xi} \quad \forall i<\xi\left(\operatorname{Prog}\left[F_{i},<_{i}, Q\right] \longrightarrow A_{i} \subseteq Q\right)$,

for each formula $Q$ in $\operatorname{ID}_{\xi}^{\mathrm{i}}(\mathfrak{X})$.

And further $\operatorname{ID}_{\xi}^{i}(\mathfrak{U})$ has the following $(\mathrm{TI})_{\xi}$ going beyond the Heyting's arithmetic :

$(\mathrm{TI})_{\xi} \quad \forall i<\xi(\forall j<i Q(j) \longrightarrow Q(i)) \longrightarrow \forall i<\xi Q(i)$

for each formula $Q$ in $\operatorname{ID}_{\xi}^{\mathrm{i}}(\mathfrak{X})$.

The intended meanings of $A_{i}(\mu)$ and $F_{j}(\nu)$ are that $\mu$ is $i$-accessible and $\nu$ is a $j$-fan in the sense of introduction.

The following proposition is easily verified: 
PROPOSITION 1. The following formulae are all derivable in $\mathrm{ID}_{\xi}^{\mathrm{i}}(\mathfrak{U})$ :

1.1. $\forall i<\xi\left(A_{i} \cong F_{i}\right)$;

1.2. $\forall i<\xi \forall \mu\left(A_{i}(\mu) \rightarrow \forall \nu<{ }_{i} \mu\left(F_{i}(\nu) \rightarrow A_{i}(\nu)\right)\right)$;

1.3. $\forall i \leqq \xi \forall \mu \forall \nu\left(\mu \equiv \nu \wedge F_{i}(\mu) \rightarrow F_{i}(\nu)\right)$;

1.4. $\forall i<\xi \forall \mu \forall \nu\left(\mu \equiv \nu \wedge A_{i}(\mu) \rightarrow A_{i}(\nu)\right)$;

1.5. $\forall i<\xi \forall \mu\left(\forall \nu\right.$ (' $\nu$ is a component of $\left.\left.\mu^{\prime} \rightarrow A_{i}(\nu)\right) \rightarrow A_{i}(\mu)\right)$.

LEMMA 2. Let $\cap_{k<i} A_{k}(\mu)$ be the formula $\forall k<i A_{k}(\mu)$. Then $\forall i \leqq \xi\left(\forall j<i\left(A_{j} \subseteq \cap_{k<j} A_{k}\right) \rightarrow \operatorname{Prog}\left[F_{i},<_{i}, \cap_{k<i} A_{k}\right]\right)$ is derivable in $\operatorname{ID}_{\xi}^{\mathrm{i}}(\mathfrak{U})$.

PROOF.

2.1. The case $i=0$. Trivial.

2.2. The case $\operatorname{Suc}(i)$.

Put

then

$$
i_{0}=i \ominus 1
$$

Assume that

$$
i=i_{0} \oplus 1 \text { and } i_{0}<i
$$

$$
\forall j<i\left(A_{j} \subseteq \cap_{k \prec j} A_{k}\right),
$$

then we have

Now we have to show

$$
\bigcap_{k<i} A_{k}=A_{i_{0}} .
$$

$$
\operatorname{Prog}\left[F_{i_{0} \oplus 1},<_{i_{0} \oplus 1}, A_{i_{0}}\right] .
$$

But the proof of lemma 26.32 in [9] can be regarded as the proof of $\operatorname{Prog}\left[F_{i_{0} \oplus 1},<_{i_{0} \oplus 1}, A_{i_{0}}\right]$ in $\operatorname{ID} \mathfrak{\xi}(\mathfrak{X})$.

2.3. The case $\operatorname{Lim}(i)$.

We can read the proof of lemma 26.33 in [9] as the proof of this case in $\operatorname{ID}_{\xi}^{\mathrm{i}}(\mathfrak{A})$.

Lemma 3. Let $\bar{A}$ be $\cap_{i<\xi} A_{i}$. Then $\operatorname{Prog}\left[F_{\xi},<_{\xi}, \bar{A}\right]$ is derivable in $\operatorname{ID}_{\xi}^{\mathbf{i}}(\mathfrak{A})$.

PROOF.

From $(A .2)_{\xi}$ we have

$$
\forall j<\xi\left(\operatorname{Prog}\left[F_{j},<_{j}, \cap_{k<j} A_{k}\right] \longrightarrow A_{j} \subseteq \cap_{k<j} A_{k}\right) .
$$

Hence it follows from lemma 2 that

$$
\forall i \leqq \xi\left(\forall j<i \operatorname{Prog}\left[F_{j},<_{j}, \cap_{k<j} A_{k}\right] \longrightarrow \operatorname{Prog}\left[F_{i},<_{i}, \cap_{k<i} A_{k}\right]\right) .
$$

It follows from this and $(\mathrm{TI})_{\xi}$ that

$$
\forall i<\xi \operatorname{Prog}\left[F_{i},<_{i}, \cap_{k<i} A_{k}\right],
$$


and

$$
\forall i \prec \xi \operatorname{Prog}\left[F_{i},<_{i}, \cap_{k<i} A_{k}\right] \longrightarrow \operatorname{Prog}\left[F_{\xi},<_{\xi}, \bar{A}\right] .
$$

Therefore the assertion follows.

LEMMA 4. $\forall \mu<_{\xi}(\xi, 0)\left(F_{\xi}(\mu) \rightarrow \bar{A}(\mu)\right)$ is derivable in $\operatorname{ID}_{\xi}^{\mathrm{i}}(\mathfrak{2})$.

PROOF.

Let $R_{i}(\nu)$ be the formula :

$$
\forall \mu<_{\xi}(i, \nu)\left(F_{\xi}(\mu) \longrightarrow \bar{A}(\mu)\right) .
$$

Firstly we will prove the following 4.1.:

4.1. $\forall i<\xi\left(R_{i}(0) \longrightarrow \operatorname{Prog}\left[F_{i},<_{i}, R_{i}\right]\right)$.

For this, suppose that $i\left\langle\xi, R_{i}(0), F_{i}(\rho), \forall \nu<_{i} \rho\left(F_{i}(\nu) \rightarrow R_{i}(\nu)\right), \mu<_{\xi}(i, \rho)\right.$ and $F_{\xi}(\mu)$.

Now we want to show that $\bar{A}(\mu)$. We may assume $\mu$ is connected by proposition 1.5 .

Furthermore we may assume

$$
(i, 0) \leqq \xi \mu<_{\xi}(i, \rho)
$$

by the assumptions $R_{i}(0)$ and $\mu_{<_{\xi}}(i, \rho)$. Therefore $\mu$ must be of the form $\left(i, \mu^{\prime}\right)$. $i<\xi$ and $\left(i, \mu^{\prime}\right)<_{\xi}(i, \rho)$ imply $\left.\mu^{\prime}<_{i} \rho . \quad F_{\xi}\left(i, \mu^{\prime}\right)\right)$ implies $A_{i}\left(\mu^{\prime}\right)$. It follows from proposition 1.1. that $F_{i}\left(\mu^{\prime}\right)$. It follows from these and the assumption $\forall \nu<_{i} \rho\left(F_{i}(\nu) \rightarrow R_{i}(\nu)\right)$ that $R_{i}\left(\mu^{\prime}\right)$, i.e.,

$$
\forall \lambda<_{\xi} \mu\left(F_{\xi}(\lambda) \longrightarrow \bar{A}(\lambda)\right) \text {. }
$$

It follows from this and lemma 3 that $\bar{A}(\mu)$.

4.1. and $(A .2)_{\xi}$ imply that

$$
\forall i \prec \xi\left(R_{i}(0) \longrightarrow A_{i} \leqq R_{i}\right) .
$$

Since for some primitive recursive function $f$, we have :

$$
\forall i<\xi \forall \mu\left(\mu<_{\xi}(i \oplus 1,0) \wedge F_{\xi}(\mu) \longrightarrow \mu<_{\xi}(i, f(i, \mu)) \wedge A_{i}(f(i, \mu))\right)
$$

we have the following 4.2. :

4.2. $\forall i<\xi\left(R_{i}(0) \longrightarrow R_{i \oplus 1}(0)\right)$.

On the other hand, $R_{0}(0)$ and $\forall i<\xi\left(\operatorname{Lim}(i) \wedge \forall j<i R_{j}(0) \rightarrow R_{i}(0)\right)$ clearly hold. Hence from $(\mathrm{TI})_{\xi}$ we have :

4.3. $\forall i<\xi R_{i}(0)$.

If $\operatorname{Lim}(\xi)$ holds, then the assertion follows from 4.3. Assume that $\operatorname{Suc}(\xi)$, i.e., 
$\xi=(\xi \ominus 1) \oplus 1$. By 4.3. and 4.2. we have $R_{\xi \ominus_{1}}(0), R_{\xi \ominus_{1}}(0) \rightarrow R_{\xi}(0)$, hence also $R_{\xi}(0)$.

TI $[X, R, Y, \mu]$ abbreviates the formula:

$$
X(\mu) \wedge(\operatorname{Prog}[X, R, Y] \longrightarrow \forall \nu(R(\nu, \mu) \wedge X(\nu) \longrightarrow Y(\nu))
$$

and TI $[X, R, \mu]$ denotes the schema $\{\text { TI }[X, R, Q, \mu]\}_{Q}$. Namely, 'TI $[X, R, \mu]$ is derivable in $\operatorname{ID}_{\xi}^{\mathrm{i}}(\mathfrak{U})$ ' means that $\operatorname{TI}[X, R, Q, \mu]$ is derivable in $\operatorname{ID}_{\xi}^{\mathrm{i}}(\mathfrak{X})$ for every formula $Q$ in $\operatorname{ID}_{\xi}^{\mathbf{i}}(\mathfrak{U})$.

Lemma 5. TI $\left[F_{\xi},<_{\xi},(\xi, 0)\right]$ is derivable in $\operatorname{ID}_{\xi}^{\mathrm{i}}(\mathfrak{A})$.

PROOF.

5.1. The case $\operatorname{Lim}(\xi)$.

For each formula $Q$, let $Q_{i}(\mu)$ be the formula :

$$
\mu<_{\xi}(i, 0) \longrightarrow Q(\mu) \text {. }
$$

Since $\mu<_{\xi}(i, 0)$ implies that $\mu$ has no $j$-section for all $j \geqq i$, the following is easily verified :

$$
\mu<_{\xi}(i, 0) \longrightarrow\left(\nu<_{i} \mu \wedge F_{i}(\nu) \wedge \nu<_{\xi}(i, 0) \longleftrightarrow \nu<_{\xi} \mu \wedge F_{\xi}(\nu)\right) .
$$

It follows from this that:

$$
\operatorname{Prog}\left[F_{\xi},<_{\xi}, Q\right] \longrightarrow \forall i<\xi \operatorname{Prog}\left[F_{i},<_{i}, Q_{i}\right] .
$$

This and $(A .2)_{\xi}$ imply that:

That is,

$$
\operatorname{Prog}\left[F_{\xi},<_{\xi}, Q\right] \longrightarrow \forall i<\xi\left(A_{i} \cong Q_{i}\right) .
$$

$$
\operatorname{Prog}\left[F_{\xi},<_{\xi}, Q\right] \longrightarrow \forall i<\xi \forall \mu<_{\xi}(i, 0)\left(A_{i}(\mu) \longrightarrow Q(\mu)\right.
$$

Thus by lemma 4 we have the assertion.

5.2. The case $\operatorname{Suc}(\xi)$.

We have easily the following 5.2.1. :

5.2.1. $\forall \mu \forall \nu\left(\nu<_{\xi} \mu<_{\xi}(\xi, 0) \longrightarrow \nu<_{\xi \ominus_{1}} \mu\right)$.

Put

$$
R(\mu):=\mu<_{\xi}(\xi, 0) \longrightarrow Q(\mu),
$$

then we have the following 5.2 .2 . by 5.2.1. :

5.2.2. $\operatorname{Prog}\left[F_{\xi},<_{\xi}, Q\right] \longrightarrow \operatorname{Prog}\left[F_{\xi \Theta_{1}},<_{\xi \ominus_{1}}, R\right]$.

It follows from 5.2.2. and $(A .2)_{\xi}$ that :

$$
\operatorname{Prog}\left[F_{\xi},<_{\xi}, Q\right] \longrightarrow \forall \mu<_{\xi}(\xi, 0)\left(A_{\xi \Theta_{1}}(\mu) \longrightarrow Q(\mu)\right) .
$$


Thus by lemma 4 we have the assertion.

Let $\bar{n}$ be the numeral corresponding to $n$ for each natural number $n$. Let $\lambda x . \xi(x, 0)$ be the primitive recursive function defined by:

$$
\xi(0,0)=0, \quad \xi(x+1,0)=(\xi, \xi(x, 0)) .
$$

Next, we will show that TI $\left[F_{\xi},<_{\xi}, \xi(\bar{n}, 0)\right]$ implies TI $\left[F_{\xi},<_{\xi}, \xi(\overline{n+1}, 0)\right]$ for $n \geqq 1$, following Gentzen [5].

Let $\lambda \mu \nu . \mu+\xi_{\nu}$ be a primitive recursive function such that:

$$
\mu \equiv 0 \longrightarrow \mu+\xi_{\nu}=\nu+{ }^{\xi} \mu=\nu \text {. }
$$

Suppose $\mu \neq 0, \nu \not \equiv 0$ and

$$
\begin{aligned}
& \mu \equiv \mu_{1} \# \cdots \# \mu_{m}, \quad \mu_{1} \geqq \cdots \mu_{\xi} \geqq \mu_{m} \equiv 0, \\
& \nu \equiv \nu_{1} \# \cdots \# \nu_{n}, \quad \nu_{1} \geqq \cdots \xi \geqq \nu_{n} \neq 0 .
\end{aligned}
$$

Let $l$ be the number such that

Then

$$
0 \leqq l \leqq m \quad \text { and } \quad \mu_{l \xi} \geqq \nu_{1 \xi}>\mu_{l+1} .
$$

$$
\mu+\xi_{\nu}=\mu_{1} \# \cdots \# \mu_{l} \# \nu_{1} \# \cdots \# \nu_{n} \text {. }
$$

LEMMA 6. For each formula $Q$, let $t[Q](\mu)$ be the formula $\forall \rho\left(F_{\xi}(\rho) \rightarrow\left(\forall \nu<_{\xi} \rho\left(F_{\xi}(\nu) \rightarrow Q(\nu)\right) \rightarrow \forall \nu<_{\xi} \rho+\xi \mu\left(F_{\xi}(\nu) \rightarrow Q(\nu)\right)\right)\right)$. Then $\operatorname{Prog}\left[F_{\xi},<_{\xi}, Q\right] \rightarrow \operatorname{Prog}\left[F_{\xi},<_{\xi}, t[Q]\right]$ is derivable in $\operatorname{ID}_{\xi}^{\mathrm{i}}(\mathfrak{H})$.

PROOF.

Obvious.

LEMMA 7. For each formula $Q$, let $s[Q](\mu)$ be the formula $t[Q]((\xi, \mu))$, i.e., $\forall \rho\left(F_{\xi}(\rho) \longrightarrow\left(\forall \nu<_{\xi} \rho\left(F_{\xi}(\nu) \longrightarrow Q(\nu)\right) \longrightarrow \forall \nu<_{\xi} \rho+\xi(\xi, \mu)\left(F_{\xi}(\nu) \longrightarrow Q(\nu)\right)\right)\right)$

Then

$$
\operatorname{Prog}\left[F_{\xi},<_{\xi}, Q\right] \longrightarrow \operatorname{Prog}\left[F_{\xi},<_{\xi}, s[Q]\right]
$$

is derivable in $\operatorname{ID}_{\xi}^{\mathrm{i}}(\mathfrak{2})$.

ProOF.

By induction on $x$, we have:

7.1. $F_{\xi}(\lambda) \wedge s[Q](\lambda) \wedge F_{\xi}(\rho) \wedge \forall \nu<_{\xi} \rho\left(F_{\xi}(\nu) \longrightarrow Q(\nu)\right) \longrightarrow$

$$
\longrightarrow \forall x \forall \nu<_{\xi} \rho+\xi(\xi, \lambda) \cdot x\left(F_{\xi}(\nu) \longrightarrow Q(\nu)\right)
$$

where $\mu \cdot x=\mu \# \cdots * \mu(x$ times $)$. 
Since we can define primitive recursive functions $f$ and $g$ such that:

$$
\begin{aligned}
\mu \neq 0 & \wedge \nu<_{\xi} \rho+\xi(\xi, \mu) \wedge F_{\xi}(\nu) \longrightarrow F_{\xi}(f(\nu, \rho, \mu)) \wedge f(\nu, \rho, \mu)<_{\xi} \mu \wedge \\
& \wedge \nu<_{\xi} \rho+\xi(\xi, f(\nu, \rho, \mu)) \cdot g(\nu, \rho, \mu),
\end{aligned}
$$

it follows from 7.1. that:

7.2. $\mu \neq 0 \wedge F_{\xi}(\mu) \wedge \forall \lambda<_{\xi} \mu\left(F_{\xi}(\lambda) \longrightarrow s[Q](\lambda)\right) \longrightarrow s[Q](\mu)$.

By lemmata 5 and 6, we have:

7.3. $\operatorname{Prog}\left[F_{\xi},<_{\xi}, Q\right] \longrightarrow s[Q](0)$.

7.2. and 7.3. imply that:

$$
\operatorname{Prog}\left[F_{\xi},<_{\xi}, Q\right] \longrightarrow \operatorname{Prog}\left[F_{\xi},<_{\xi}, s[Q]\right] .
$$

From lemmata 5 and 7 , we have the following lemma by metainduction on $n$.

LEMMA 8. TI $\left[F_{\hat{\xi}},<_{\xi}, \xi(\bar{n}, 0)\right]$ is derivable in $\operatorname{ID}_{\xi}^{\mathrm{i}}(\mathfrak{A})$ for each natural number $n$.

THEOREM. $A_{0}(\lceil\mu\urcorner)$ is derivable in $\operatorname{ID}_{\xi}^{\mathrm{i}}(\mathfrak{U})$ for each o.d. $\mu$ from $O^{*}(I, 1)$, where $\lceil\mu\rceil$ is the gödelnumber of $\mu$.

PROOF.

For some primitive recursive function $f$, we have in PRA $\nu \leqq_{0} \xi(f(\nu), 0)$. By lemmata 3 and 8 we have $\bar{A}(\xi(f(\ulcorner\mu\urcorner), 0))$ in $\operatorname{ID}_{\xi}^{\mathrm{i}}(\mathfrak{U})$. In particular $A_{0}(\xi(f(\ulcorner\mu\rceil), 0))$. Hence from proposition 1.2. $A_{0}(\ulcorner\mu\urcorner)$ is derivable in $\operatorname{ID}_{\xi}^{\mathrm{i}}(\mathfrak{A})$.

\section{REMARKS.}

1. Let $\mathrm{T}^{\mathrm{i}}$ be the theory $\operatorname{ID}_{\xi}^{\mathrm{i}}(\mathfrak{A})$ and $\operatorname{Prov}_{\mathrm{T}^{\mathrm{i}}}$ be a canonical proof-predicate for $\mathrm{T}^{\mathrm{i}}$. Then we have constructed a primitive recursive function $p$ such that:

PRA proves that ' $x$ is an o.d. from $O^{*}(I, 1)$ ' $\longrightarrow \operatorname{Prov}_{\mathrm{T}^{\mathrm{i}}}\left(\not p(x), \Gamma A_{0}(\dot{x}) 7\right)$, where $\left\lceil A_{0}(\dot{x})\right\rceil$ is a term whose value is the gödelnumber of $A_{0}(\bar{n})$ when the numeral $\bar{n}$ is substituted for the variable $x$.

2. Let the order type of $\prec$ be 2 or $\omega+1, T$ be the classical version of $T^{i}$ and $\mathrm{T}^{*}$ be the subsystem $(\mathrm{BI})$ or $\left(\Pi_{1}^{1}-\mathrm{CA}\right)+(\mathrm{BI})$ of classical analysis, respectively. Then by the well-known translation * (cf. [4].), we have

and also

$$
\mathrm{T} \vdash A_{0}(\mu) \quad \text { implies } \mathrm{T}^{*} \vdash A_{0}^{*}(\mu)
$$

$$
\mathrm{T}^{*} \vdash A_{0}^{*}(\mu) \longrightarrow \mathrm{TI}_{<0}[\mu]
$$

where $\mathrm{TI}_{<0}[\mu]$ is the formula 


$$
\forall X\left(\forall \nu<_{0} \mu\left(\forall \rho<_{0} \nu X(\rho) \longrightarrow X(\nu)\right) \longrightarrow \forall \nu<_{0} \mu X(\nu)\right) .
$$

Hence from the remark 1, we have:

PRA proves that ' $x$ is an o.d. from $O^{*}(I, 1)$ ' $\rightarrow \operatorname{Prov}_{\mathrm{T}^{*}}\left(p^{*}(x), \mathrm{TI}_{<_{0}}[\dot{x}]\right)$

for some primitive recursive function $p^{*}$.

On the other hand, we will prove in [1] the consistency of $(\mathrm{BI}),\left(\Pi_{1}^{1}-\mathrm{CA}\right)+$ (BI) by the accessibility of $O(2,1), O(\omega+1,1)$ with respect to $<_{0}$, respectively.

3. From the remark 2, we have

$$
\left|\mathrm{ID}_{\omega}^{\mathrm{i}}\right|=\left|\mathrm{ID}_{\omega}\right|=\left|\left(\Pi_{1}^{1}-\mathrm{CA}\right)+(\mathrm{BI})\right|=|O(\omega+1,1)|_{<0}
$$

where $\left|\mathrm{ID}_{\omega}^{\mathrm{i}}\right|$ denotes the order type of the least unprovable recursive wellordering in $\mathrm{ID}_{\omega}^{\mathrm{i}}$, etc., and $|O(\omega+1,1)|_{<0}$ denotes the order type of the system $O(\omega+1,1)$ with respect to $<_{0}$.

Following Buchholz and Pohlers [2], and Pohlers [8] the common ordinal equals to $\Theta \varepsilon_{\Omega_{\omega^{+}}} 0$. Thus we have indirectly:

$$
|O(\omega+1,1)|_{<0}=\Theta \varepsilon_{\Omega_{\omega^{+1}}} 0 .
$$

This is an analogue to the fact:

$$
|O(n+1,1)|_{<0}=\Theta \varepsilon_{\Omega_{n}+1} 0 \quad \text { for every } n \text { such that } 1 \leqq n<\omega .
$$

But note that the latter was established directly in Levitz [7] and Buchholz and Schütte [3].

4. By [2] and [8]

and

$$
|\mathrm{ID} \bar{\xi}|=\left|\mathrm{ID}_{\xi}\right|=\Theta \varepsilon_{\Omega_{\xi}+1} 0 \quad \text { for } \quad \xi<\Theta \Omega_{\Omega_{1}} 0,
$$

$$
\left|\mathrm{ID}_{<\xi}^{\mathrm{i}_{<\xi}}\right|=\left|\mathrm{ID} \mathrm{D}_{<\xi}\right|=\Theta \Omega_{\xi} 0=\sup _{\zeta<\xi} \Theta \varepsilon_{\Omega_{\Omega^{+1}}} 0 \quad \text { for limit } \quad \xi \leqq \Theta \Omega_{\Omega_{1}} 0 .
$$

On the other hand, for limit $\xi$ and $\zeta<\xi$, the subsystem $\left\{\mu \in O(\xi, 1): \mu_{0}(\zeta+1,0)\right\}$ of $O(\xi, 1)$ is nothing but $O(\zeta+1,1)$,

Hence we have:

$$
|O(\xi, 1)|_{<0}=\sup _{\zeta<\xi}|O(\zeta+1,1)|_{<0} \quad \text { for limit } \xi .
$$

So one may conjecture that

$$
\begin{aligned}
& |O(\xi+1,1)|_{<0}=\Theta \varepsilon_{\Omega_{\xi^{+1}}} 0, \\
& |O(\xi, 1)|_{<0}=\Theta \Omega_{\xi} 0, \quad \xi ; \text { limit, }
\end{aligned}
$$

for appropriately small $\xi$.

But we have not verified this conjecture in any way. 


\section{References}

[1] Arai, T., A subsystem of classical analysis proper to Takeuti's reduction method for $\Pi_{1}^{1}$-analysis. in preparation.

[2] Buchholz, W. and Pohlers, W., Provable wellordering of formal theories for transfinitely iterated inductive definitions. J. Symbolic Logic 43 (1978), 118-125.

[3]. - and Schütte, K., Die Beziehungen $z$ wishen den Ordinalzahlsystemen $\Sigma$ und $\bar{\Theta}(\omega)$. Archiv für mathematishe Logik und Grndlagenforshung 17 (1975), 179190.

[4] Feferman, S., Formal theories for transfinite iterations of generalized inductive definitions and some subsystems of analysis. Intuitionism and proof theory (Kino, Myhill and Vesley, Editors), North-Holland, Amsterdam (1970), 303-326.

[5] Gentzen, G., Beweisbarkeit und Unbeweisbarkeit von Anfangsfällen der transfiniten Induktion in der reinen Zahlentheorie. Mathematishe Annalen 119, No. 1 (1943), 140-161.

[6]. Kreisel, G., Review. Zentralblatt für Mathematik und ihre Grenzgebiete 106 (1964), 237-238.

[7] Levitz, H., On the relationship between Takeuti's ordinal diagrams $O(n)$ and Schütte's system of ordinal notations $\Sigma(n)$. Intuitionism and proof theory (Kino, Myhill and Vesley, Editors), North-Holland, Amesterdam (1970), 377405.

[8] Pohlers, W., Ordinals connected with formal theories for transfinitely iterated inductive definitions. J. Symbolic Logic 43 (1978), 161-182.

[9] Takeuti, G., Proof theory. North-Holland, Amsterdam (1975).

Institute of Mathematics

University of Tsukuba

Sakura-mura, Niihari-gun

Ibaraki, 305 Japan 